\title{
Rehabilitación ecológica en el noreste patagónico: Supervivencia y reclutamiento de especies nativas en suelos con diferentes texturas
}

\author{
Juan M. Zeberio ${ }^{1,3, \bigotimes}$ \& Carolina A. Pérez ${ }^{2}$ \\ ${ }^{1}$ Universidad Nacional de Río Negro. Sede Atlántica. Centro de Estudios Ambientales desde la Norpatagonia (CEANPa). \\ ${ }^{2}$ Laboratorio de Investigación de Sistemas Ecológicos y Ambientales (LISEA). FCAyF - FCNyM. Universidad Nacional de \\ La Plata. ${ }^{3}$ Consejo Nacional de Investigaciones Científicas y Técnicas (CONICET).
}

\begin{abstract}
Resumen. Los suelos del noreste patagónico presentan una marcada heterogeneidad, dada principalmente por su textura. En esta región se observan fuertes procesos de degradación. El objetivo fue estimar la supervivencia de especies nativas en ensayos de rehabilitación ecológica en áreas degradadas, sobre suelos de diferente textura, y evaluar el reclutamiento alrededor de los ejemplares trasplantados sobrevivientes. Los estudios se realizaron en tres sitios con historia similar de uso, con suelos franco, franco-arenoso y arenoso. En cada sitio se instalaron tres parcelas de $100 \mathrm{~m}^{2}$, excluidas del pastoreo del ganado. Se trasplantaron individuos de especies leñosas (Condalia microphylla, Geoffroea decorticans, Prosopis flexuosa y Schinus johnstonii) y herbáceas (Nassella longiglumis, Nassella tenuis, Pappophorum caespitosum y Piptochaetium napostaense). El reclutamiento se estimó en parcelas de $50 \times 50 \mathrm{~cm}$, dejando al ejemplar trasplantado sobreviviente en el centro. Las estimaciones fueron realizadas cuatro años después del trasplante. El reclutamiento se categorizó en especies leñosas forrajeras y no forrajeras, y herbáceas anuales o perennes forrajeras y no forrajeras. La textura del suelo parece no influir en la supervivencia de las especies herbáceas trasplantadas, pero sí en una leñosa (S. johnstonii), que fue significativamente mayor en suelos franco-arenosos. La riqueza de especies reclutadas fue similar entre sitios, excepto para las herbáceas anuales no forrajeras, que fueron mayores en suelos francos y franco-arenosos. No se detectó reclutamiento de leñosas trasplantadas, pero sí de otras leñosas nativas, mientras que el reclutamiento de herbáceas perennes fue sólo de las trasplantadas. La densidad no evidenció correspondencia con la textura del suelo, excepto para las especies herbáceas anuales forrajeras y no forrajeras, cuyas densidades fueron mayores en sitios franco y franco-arenoso, respectivamente. La mayoría de las especies seleccionadas son aptas para proyectos de rehabilitación ecológica. La provisión de semillas de cada sitio tendría una importancia preponderante en la riqueza y la densidad del reclutamiento.
\end{abstract}

[Palabras clave: degradación, revegetación, tierras secas, especies nativas, leñosas, herbáceas]

\begin{abstract}
Aвstract. Ecological rehabilitation in northeastern Patagonia: Survival and recruitment of native species in soils with different textural characteristics. In the northeastern Patagonian region, soils show marked heterogeneity, mainly due to their texture. It is also observed strong degradation processes in this region. The objective was to estimate survival rate of native species in ecological rehabilitation trials in degraded areas, on soils of different textures, and evaluate recruitment around surviving transplanted individuals at each site. Studies were performed in three sites that had a similar land use history, in loamy, sandy loam and sandy soils. At each site, three $100 \mathrm{~m}^{2}$ plots, excluded from cattle grazing were installed. Individuals of woody (Condalia microphylla, Geoffroea decorticans, Prosopis flexuosa y Schinus johnstonii) and herbaceous (Nassella longiglumis, Nassella tenuis, Pappophorum caespitosum and Piptochaetium napostaense), native species were transplanted. Recruitment was estimated in 50x50 cm plots, leaving the surviving transplanted individual in the center. Survival rate and recruitment were estimated four years after transplantation. Recruitment was categorized into forage and non-forage woody plants and annual or perennial, forage, and non-forage herbaceous plants. Soil texture seems did not influence in survival of herbaceous transplanted species, but it did in a woody plant (S. johnstonii), that was significantly higher in sandy loam soils. Richness of recruited woody and herbaceous species was similar between sites, except for annual non-forage herbaceous plants, being richer in loam and sandy loam sites. Transplanted woody species recruitment was not detected, but recruitment of other native woody species was recorded, and the recruitment of perennial herbaceous species was only of the transplanted species. Recruitment density did not show correspondence with soil texture, except for forage and non-forage annual herbaceous species, that was higher at the loam and sandy loam sites, respectively. Most of the selected species presented survival rates that make them suitable for ecological rehabilitation projects. Seed supply of each site would have a preponderant importance to recruitment recovery.
\end{abstract}

[Keywords: degradation, revegetation, dry lands, woody species, herbaceous species]

Editora asociada: Vanessa Lencinas

jmzeberio@unrn.edu.ar
Recibido: 23 de Diciembre de 2020

Aceptado: 8 de Julio de 2021 


\section{INTRODUCCIÓN}

La heterogeneidad de los suelos es un factor importante en ecosistemas áridos y semiáridos de todo el mundo, donde la vegetación se organiza en parches sobre una matriz de suelo desnudo (Aguiar and Sala 1999). Los suelos son temporal y espacialmente variables, y su heterogeneidad influye sobre la estructura y los procesos funcionales de la vegetación (Maestre et al. 2003). La degradación del suelo afecta a las comunidades vegetales y a los procesos ecosistémicos (Tongway and Hindley 2005; Maestre et al. 2016). En términos generales, la degradación de los sistemas áridos y semiáridos está muy asociada al uso productivo que el ser humano hace de ellos (Cortina et al. 2011). Cuando los ecosistemas se degradan, disminuye la captura de recursos indispensables para el desarrollo de las plantas, se empobrece la funcionalidad y la estructura de la vegetación, y también disminuye su capacidad para prestar servicios ecosistémicos como proveer forraje, retener partículas de suelo superficial para evitar la erosión y capturar $\mathrm{CO}_{2}$ atmosférico (Tongway and Hindlley 2005).

En ecosistemas áridos y semiáridos, la disponibilidad de agua en el suelo controla la germinación y el establecimiento posterior de las plántulas (Maestre et al. 2003). En estos sistemas, el agua disponible muestra un alto grado de heterogeneidad a lo largo de gradientes temporales de precipitación, marcados por la estacionalidad y la variabilidad inter- e intra-anual (Le Houerou et al. 1989; Seghieri et al. 1997). A su vez, la compactación del suelo superficial, la presencia de costras físicas y fragmentos de roca tienen un rol importante en la dinámica de la escorrentía y de la infiltración (Valentin and Bresson 1992). Otras propiedades del suelo, como el porcentaje de materia orgánica y el contenido de arena, también son relevantes para determinar la capacidad de retención de agua del suelo (Ward and Robinson 1990). Existe una correlación negativa entre el contenido de arena de los suelos superficiales, el contenido de materia orgánica y la cobertura vegetal (Buschiazzo et al. 1991, 2004).

La heterogeneidad espacial de los suelos suele ser un elemento clave a tener en cuenta para rehabilitar ecosistemas degradados (Whisenant 1999). Según Aronson et al. (1993) y Hobbs and Cramer (2008), la rehabilitación ecológica consiste en realizar tareas sobre un sistema natural con la finalidad de devolverle alguno de los atributos estructurales y funcionales perdidos. Estas acciones incluyen eliminar el disturbio causante de la degradación y repoblar con organismos vegetales para favorecer la colonización de áreas de suelo desnudo. Este repoblamiento se puede promover tanto a partir de semillas como de renovales de especies nativas o exóticas (Dalmasso 2010; Landi and Renison 2010; Torres et al. 2015), como así también aplicando enmiendas al suelo para modificar sus características físicas y químicas (DelgadoBaquerizo et al. 2013), roturando la superficie para eliminar el encostramiento superficial y favorecer la infiltración (Gasch et al. 2014), o a partir de combinaciones de estas técnicas. El repoblamiento con especies vegetales suele ser la técnica más frecuente (Peláez et al. 1996; Maestre et al. 2001; Dalmasso 2010; Plaza Behr et al. 2016). Algunas de las especies empleadas para este fin suelen ser de la familia de las leguminosas, ya que poseen un rol ecológico destacado por su capacidad de fijar nitrógeno atmosférico a través de la asociación simbiótica con bacterias del género Rhizobium (Villagra et al. 2011; López Lauenstein et al. 2012). Otras especies seleccionadas para la restauración ecológica son aquellas capaces de generar grandes cantidades de mantillo (e.g., especies del género Prosopis) o las que actúan como fuente de forraje para animales silvestres y domésticos (e.g., Nassella longiglumis, N. tenuis, Pappophorum caespitosum y Piptochaetium napostaense). Algunas especies también pueden facilitar el establecimiento de otras. En este proceso, la especie nodriza genera condiciones en el área que la circunda que favorecen la germinación y establecimiento de individuos de otras especies, ya sea por amortiguar su exposición a factores climáticos, por mejorar la fertilidad del suelo o por brindar protección frente a herbívoros (Padilla and Pugnaire 2006; Valladares and Gianoli 2007).

En el noreste de la Patagonia existe una amplia heterogeneidad ambiental generada, en parte, por las características de los suelos, que ejercen una influencia destacada sobre la composición y abundancia de la vegetación (León et al. 1998; Godagnone and Bran 2009). A escala de paisaje se observan cambios en la fisonomía de la vegetación y en el desarrollo de los suelos. Según Godagnone y Bran (2009), se destacan dos tipos de geoformas y comunidades asociadas: las terrazas fluviales mesetiformes y los paleocanales. Las primeras son áreas elevadas con vegetación baja, dominadas por especies de Zigofiláceas (Larrea sp.), que presentan suelos menos 
profundos que los paleocanales. Estos últimos se corresponden con antiguas áreas de drenaje, con suelos más profundos, donde dominan las especies de mayor porte, principalmente de la familia Fabaceae, que coexisten con pastos y hierbas perennes y anuales (Godagnone and Bran 2009).

Entre los años 2000 y 2007, en el noreste patagónico se observó un crecimiento fuerte de la superficie de tierras destinadas a la producción agrícola de secano (Zeberio 2018). Luego, estas tierras fueron abandonadas por causas asociadas a la sequía registrada en el área en el período 2004-2010 y la falta de rentabilidad de los campos (Zeberio et al. 2018). El abandono ocurrió sin mediar ningún plan de manejo luego del cese de las actividades, dejando a merced de los factores erosivos $\sim 30 \%$ de la superficie del suelo de la región mencionada (Abraham et al. 2016; Zeberio 2018). Hasta el momento, no se realizaron tareas de restauración o de rehabilitación ecológica en el área. Esta opción sería de importancia para que esos sistemas recuperen algunos atributos funcionales y su capacidad para brindar ciertos servicios ecosistémicos.

En los ecosistemas áridos y semiáridos donde se superaron umbrales de degradación biótica y abiótica es necesaria una intervención activa para objetivos de rehabilitación ecológica. Estos objetivos pueden alcanzarse mediante el repoblamiento con individuos de especies provenientes de germoplasma local, capaces de sobrevivir en esos ambientes degradados. Se espera, además, que las especies empleadas para la rehabilitación ecológica favorezcan procesos de reclutamiento espontáneo de individuos de su propia especie o de otras nativas Este trabajo tiene como objetivos estimar la supervivencia de especies nativas empleadas en ensayos de rehabilitación realizados en áreas degradadas que presentan suelos con diferentes características texturales, y evaluar el reclutamiento de leñosas y herbáceas en las proximidades de los individuos trasplantados. Se proponen como hipótesis que: 1) la supervivencia de especies empleadas en ensayos de rehabilitación ecológica varía según las características texturales de los suelos, siendo menor en suelos arenosos debido a su menor capacidad de retención de agua, y 2) la riqueza de especies y la densidad de individuos establecidos espontáneamente (reclutamiento) es mayor en los sitios con suelos de textura franca y franco-arenosa, en relación con su mayor capacidad de retención hídrica.

\section{Materiales y Métodos}

\section{Área de estudio}

El trabajo se desarrolló en el noreste de la Patagonia argentina (Figura 1). Morello

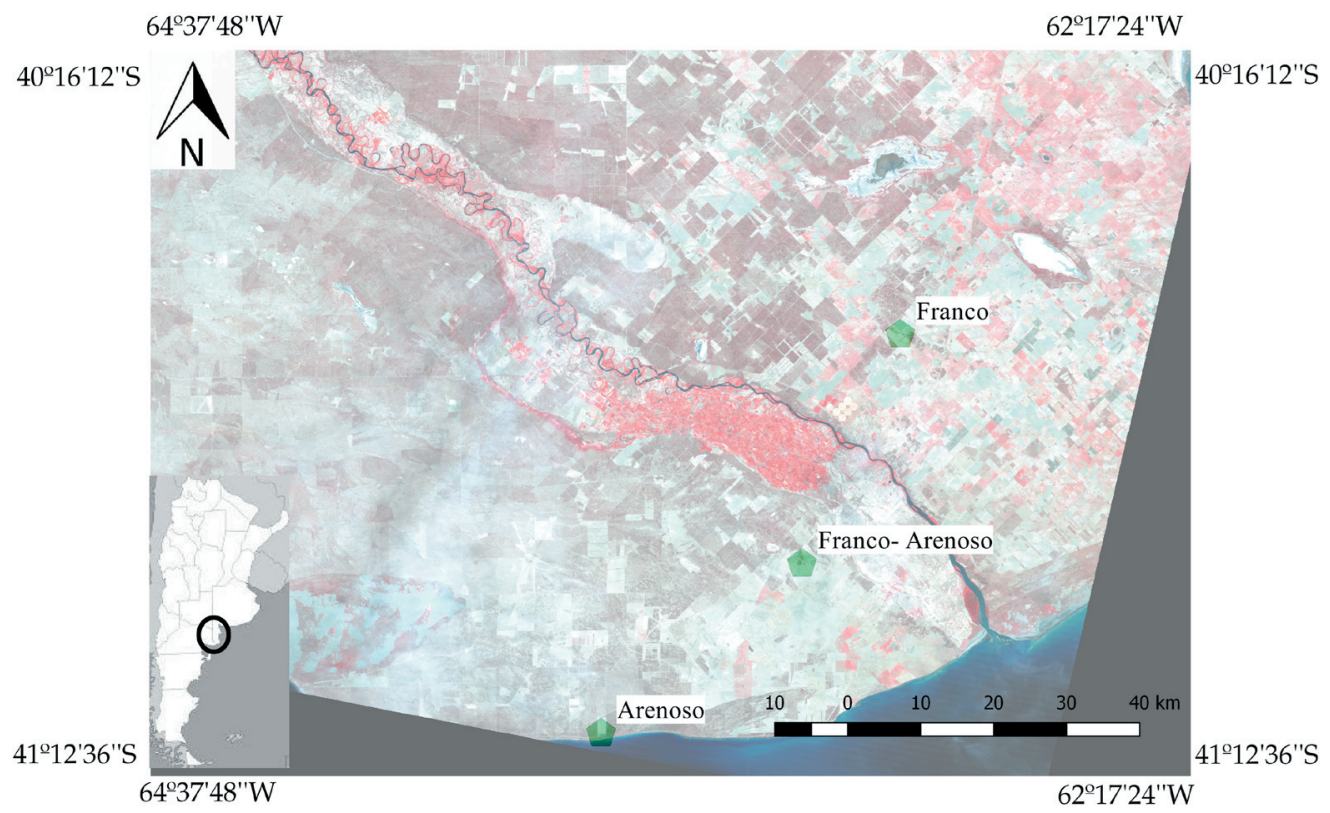

Figura 1. Ubicación de los sitios de estudio de rehabilitación ecológica.

Figure 1. Location of the ecological rehabilitation study sites. 
et al. (2012) clasificaron el área de estudio como Complejo de Planicies y Mesetas Norpatagónicas, que se encuentra dentro de la ecorregión del Monte de Llanuras y Mesetas. En ésta coexisten especies leñosas y herbáceas pertenecientes a la ecorregión del Monte y del Espinal (Morello et al. 2012; Torres Robles et al. 2015). Estructuralmente, es una estepa arbustiva de altura media en la que se encuentran elementos arbóreos más o menos aislados, acompañados por pastos y hierbas.

El clima del área de estudio es subtemplado seco de transición, con veranos cálidos e inviernos moderados y sin ningún exceso estacional de agua (De Berasategui 2004). La temperatura media anual es del orden de 14-15 ${ }^{\circ} \mathrm{C}$. La temperatura media del mes más cálido oscila entre 22 y $24^{\circ} \mathrm{C}$, y la del mes más frío, entre 7 y $8^{\circ} \mathrm{C}$. Las precipitaciones presentan un leve aumento desde la zona oeste, donde se registran las menores cantidades anuales, con una media histórica anual de $250 \mathrm{~mm}$ hacia el límite este-noreste del área de estudio, donde la precipitación media anual histórica es $311 \mathrm{~mm}$ para el período 1965- 2011 (Godagnone and Bran 2009). Durante el semestre primavera-verano, que es el de mayor importancia desde el punto de vista agronómico, las precipitaciones son irregulares por su distribución y deficitarias respecto de las demandas evaporativas del aire (De Berasategui 2004). Durante el período otoño-invierno, las lluvias se producen con mayor regularidad, lo que resulta en un régimen menos deficitario debido a las menores temperaturas, la mayor humedad relativa y la menor intensidad de los vientos (Godagnone and Bran 2009) (Figura 2). Los órdenes dominantes de suelo corresponden a los Aridisoles y Entisoles, típicos de regiones

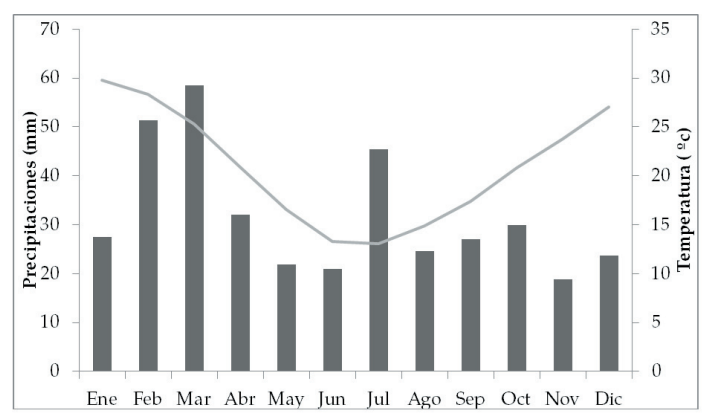

Figura 2. Climograma de precipitación media mensual y temperatura media mensual para el período 2000-2016 en el noreste de la provincia de Río Negro.

Figure 2. Climogram of mean monthly rainfall and mean monthly temperature for the period 2000-2016 in northeastern Río Negro province. áridas y semiáridas, con escaso desarrollo y bajos porcentajes de materia orgánica y nutrientes (Zeberio 2018).

En estudios previos se seleccionaron tres sitios para desarrollar tareas de rehabilitación ecológica y se determinaron sus características edáficas (Zeberio et al. 2018; Zeberio and Pérez 2020). El presente estudio se llevó adelante en esos tres sitios, que poseen características edáficas diferentes, pero una historia de uso común. Los tres fueron desmontados a tala rasa para implantar cultivos extensivos de secano por, al menos, 15 años, y luego fueron abandonados. En el sitio de textura franca, la erosión eólica ha generado una pérdida importante de la capa superficial del suelo; al momento de instalar los ensayos y tomar las muestras, tenía una profundidad de $15 \mathrm{~cm}$ hasta el contacto lítico (capa potente y homogénea de $\mathrm{CaCO}_{3}$ ). El suelo de este sitio pertenece a los Calciortides típicos, con muy bajos contenidos de materia orgánica y de nitrógeno (Tabla 1). Este sitio se ubica en la región geomorfológica de planicies interfluviales mesetiformes, en un plano alto, dentro de un sector levemente ondulado (Godagnone and Bran 2009). Los sitios sobre suelos franco-arenosos y arenosos están situados en la planicie loéssica (Godagnone and Bran 2009) y pertenecen a los Torripsamentes típicos. El sitio sobre suelo franco-arenoso presenta valores de materia orgánica, nitrógeno y fósforo más elevados que los de los otros dos sitios analizados, y no se observan limitantes en profundidad. El sitio de textura arenosa se ubica en las proximidades de la costa atlántica, con suelos poco profundos, de escaso desarrollo y excesivamente drenados, con bajos valores de materia orgánica, nitrógeno y fósforo (Tabla 1).

\section{Diseño experimental y recolección de datos}

Estudios previos a este trabajo evaluaron los remanentes de vegetación nativa del Monte en el área de estudio y determinaron las especies más frecuentes (Torres Robles et al. 2015; Zeberio et al. 2018; Zeberio and Pérez 2020). Esas especies se usaron para las tareas de rehabilitación ecológica, dado que se corroboró su ausencia en los sitios de ensayos (Zeberio 2018). Entre las leñosas se seleccionaron Condalia microphylla Cav. (Rhamnaceae), Geoffroea decorticans (Gillies ex Hook. and Arn.) Burkart (Fabaceae), Prosopis flexuosa DC. var. depressa F.A. Roig (Fabaceae) y Schinus johnstonii F.A. Barkley (Anacardiaceae); el 
Tabla 1. Características edáficas de los sitios donde se desarrollaron los ensayos de rehabilitación ecológica (Zeberio 2018; Zeberio and Pérez 2020). CE: Conductividad eléctrica (dS/m). RAS: relación de adsorción de sodio (meq/L). $\mathrm{CaCO}_{3}$ : contenido de carbonato de calcio del suelo $(\mathrm{Mg} / \mathrm{kg})$. COS: carbono orgánico del suelo $(\%)$. Nt: nitrógeno total (\%). P: fósforo (ppm). D. Ap.: densidad aparente del suelo $\left(\mathrm{g} / \mathrm{m}^{2}\right)$. Prof.: profundidad $(\mathrm{m})$.

Table 1. Trial rehabilitation soil sites characteristics (Zeberio 2018; Zeberio and Pérez 2020). CE: electrical conductivity $(\mathrm{dS} / \mathrm{m})$. RAS: sodium adsorption ratio (meq/L). CaCO3: calcium carbonate content $(\mathrm{Mg} / \mathrm{kg})$. COS: soil organic carbon (\%). Nt: total nitrogen (\%). P: phosphorus (ppm). D. Ap.: bulk density $\left(\mathrm{g} / \mathrm{m}^{2}\right)$. Prof: depth (m).

\begin{tabular}{llllllllllll}
\hline Latitud & Longitud & Textura & $\mathrm{pH}$ & $\mathrm{CE}$ & $\mathrm{RAS}$ & $\mathrm{CaCO}_{3}$ & $\mathrm{COS}$ & $\mathrm{Nt}$ & $\mathrm{P}$ & $\mathrm{D}$. Ap. & Prof. \\
\hline $40^{\circ} 39^{\prime} 30^{\prime \prime} \mathrm{S}$ & $62^{\circ} 52^{\prime} 1^{\prime \prime} \mathrm{O}$ & Franco & 7.86 & 1.78 & 13.86 & 1.67 & 0.17 & 0.03 & 2.31 & 0.43 & 0.12 \\
$41^{\circ} 1^{\prime} 4^{\prime \prime} \mathrm{S}$ & $63^{\circ} 1^{\prime} 40^{\prime \prime} \mathrm{O}$ & $\begin{array}{l}\text { Franco } \\
\text { Arenoso }\end{array}$ & 8.21 & 0.54 & 3.69 & 4.75 & 0.61 & 0.09 & 8.77 & 1.41 & 1.7 \\
$41^{\circ} 9^{\prime} 12^{\prime \prime} \mathrm{S}$ & $63^{\circ} 25^{\prime} 19^{\prime \prime} \mathrm{O}$ & Arenoso & 8.2 & 0.58 & 3.42 & 1.77 & 0.31 & 0.06 & 8.93 & 1.27 & $>2$ \\
\hline
\end{tabular}

ganado ramonea estas especies o consume sus frutos en épocas desfavorables. Entre las herbáceas, las empleadas para los ensayos fueron todas perennes, forrajeras, pertenecientes a la familia Poaceae, Nassella tenuis (Phil.) Barkworth, Nassella longiglumis (Phil.) Barkworth, Piptochaetium napostaense (Speg.) Hack. y Pappophorum caespitosum R.E. Fr.

Los individuos de las especies seleccionadas para los ensayos de rehabilitación se obtuvieron en invernáculo a partir de germoplasma local. Al cabo de 10 meses desde el momento de la siembra, y luego de efectuar prácticas de rustificación, fueron trasplantadas a campo. El trasplante se realizó durante los meses de abril y mayo del año 2012, en parcelas de $100 \mathrm{~m}^{2}$, clausuradas al pastoreo de grandes herbívoros y con mallas plásticas (tamaño de celda: $2 \times 2 \mathrm{~cm}$ ) para evitar los daños que causan las liebres. Se instalaron 3 parcelas en cada uno de los sitios seleccionados para los ensayos, distanciadas $75 \mathrm{~m}$ una de otra. En cada parcela se plantaron hileras monoespecíficas de 9 individuos de especies leñosas, excepto C. microphylla, de la cual se trasplantaron 5 individuos. Esta especie fue muy sensible a los tratamientos de rustificación, resultando insuficientes los ejemplares sobrevivientes para el ensayo de rehabilitación. Se plantaron 81 individuos de P. flexuosa, 81 de G. decorticans, 81 de S. johnstonii y 45 de C. microphylla. Todos los ejemplares de especies leñosas mantuvieron un distanciamiento de $2 \mathrm{~m}$ entre sí. De forma transversal a los individuos de especies leñosas se trasplantaron hileras monoespecíficas de 11 individuos de las especies herbáceas. Fueron plantados, en el total del ensayo, 99 individuos de las especies $N$. longiglumis, $N$. tenuis, $P$. napostaense y $P$. caespitosum. Los individuos de las especies herbáceas mantuvieron un espaciamiento aproximado de $1 \mathrm{~m}$, distribuidos según se indica en la Figura 3.

Luego de 4 años de realizado el trasplante se comparó la supervivencia de las plantas entre los sitios con suelos de diferente textura (i.e., franca, franco-arenosa y arenosa). Para evaluar el reclutamiento cerca de los individuos trasplantados sobrevivientes se empleó un marco de 50x50 cm, ubicando al individuo

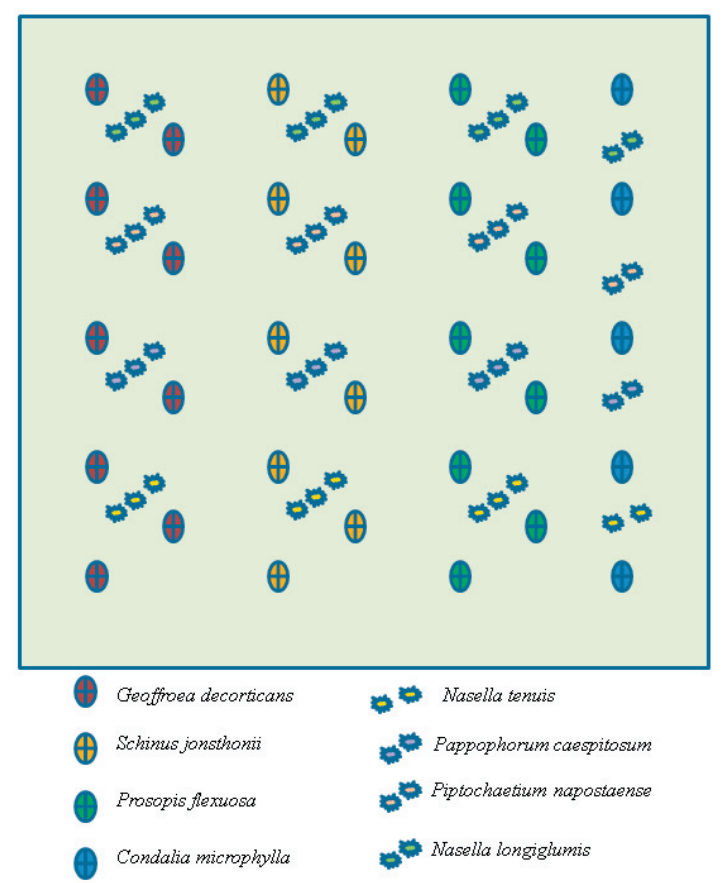

Figura 3. Diagrama del diseño experimental empleado en la reintroducción de especies nativas a campo. Los círculos representan individuos de plantas leñosas, mientras que los otros símbolos representan individuos de gramíneas perennes.

Figure 3. Diagram of the experimental design used in the reintroduction of native species to the field. The circles represent individuals of woody plants, while the other symbols represent individuals of perennial grasses. 
trasplantado en el centro. En cada sito, las plantas reclutadas fueron identificadas a nivel de especie. Se estimó la riqueza total y, según la forma de crecimiento, el ciclo de vida y el grado de preferencia del ganado. Así quedaron establecidas seis categorías: 1) herbáceas anuales forrajeras, 2) herbáceas anuales no forrajeras, 3) herbáceas perennes forrajeras, 4) herbáceas perennes no forrajera, 5) leñosas forrajera y 6) leñosas no forrajeras. Se estimó la densidad del reclutamiento (número de individuos $/ \mathrm{m}^{2}$ ) según las mismas categorías. La información relativa a las características de las especies empleadas en los tratamientos de rehabilitación y del reclutamiento (i.e., forma de crecimiento, ciclo de vida y preferencia) fue obtenida del Catálogo de Plantas Vasculares de la República Argentina (Zuloaga et al. 1994; Zuloaga and Morrone 1996, 1999) y de la Guía para el Reconocimiento de Especies de los Pastizales del Monte Oriental de la Patagonia (Kröpfl et al. 2012).

\section{Análisis de datos}

Para comparar la supervivencia de los ejemplares de las especies trasplantadas se emplearon modelos lineales generalizados con asunción de distribución binomial de los datos. Como variables predictoras se usaron el sitio y la especie. Además, se estimaron las interacciones sitio $x$ especie. Se empleo la prueba de Bonferroni para comparar las medias entre sitios y especies (Faraway 2006).

Los datos de riqueza y densidad deindividuos por metro cuadrado no cumplieron con los supuestos de normalidad y homocedasticidad. Para efectuar el análisis de la variabilidad de reclutamiento (i.e., riqueza y densidad) de las diferentes categorías de especies entre los sitios de ensayo se llevó a cabo un análisis de la varianza no paramétrico (prueba de Kruskal Wallis). Para comparar las medias y determinar la significancia estadística se utilizaron los procedimientos descriptos por Conover e Inman (1979) y Conover (1999). Todos los análisis fueron efectuados con el software INFOSTAT (Di Rienzo et al. 2018).

\section{Resultados}

\section{Supervivencia}

El análisis de la supervivencia de las especies leñosas mostró diferencias significativas entre especies $(\mathrm{F}=1.53 ; \mathrm{P}<0.0001)$, sitios $(\mathrm{F}=12.29$; $\mathrm{P}<0.001)$ y la interacción entre especies y sitios ( $\mathrm{F}=2.95 ; \mathrm{P}=0.008)$. La supervivencia varió dependiendo de la especie y el sitio considerados (Figura 4 y Tabla 3). Se destaca que la supervivencia de $S$. johnstonii en el sitio

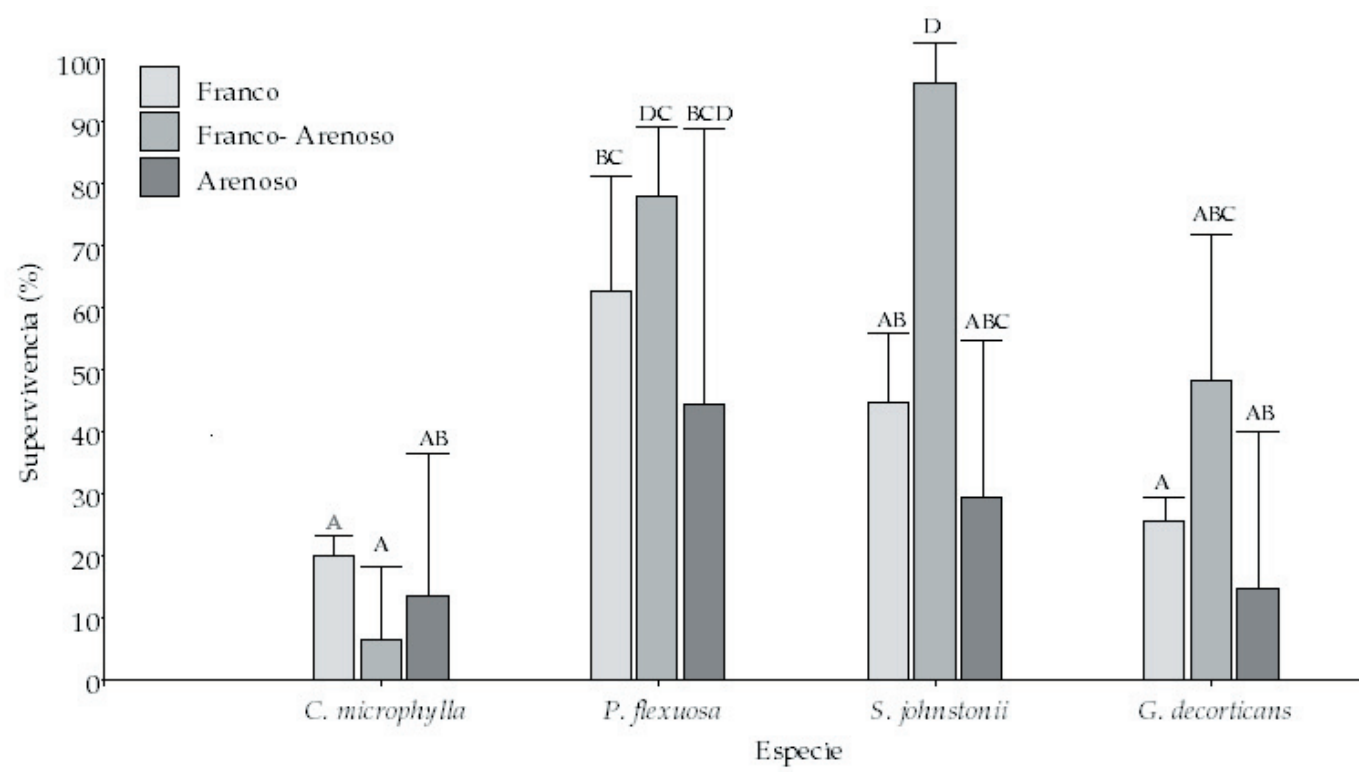

Figura 4. Supervivencia de los individuos de especies leñosas (Condalia microphylla, Prosopis flexuosa, Schinus johnstonii y Geoffroea decorticans) luego de 4 años desde el trasplante. Letras diferentes indican diferencias significativas $(\mathrm{P}<0.05)$. Barras de error representan el desvío estándar.

Figure 4. Survival of individuals of woody species (Condalia microphylla, Prosopis flexuosa, Schinus johnstonii and Geoffroea decorticans) after 4 years from transplant. Different letters indicate significant differences $(\mathrm{P}<0.05)$. Error bars represent the standard deviation. 
Tabla 2. Características de las especies reclutadas en ensayos de rehabilitación en sitios con diferente textura del suelo.

Table 2. Characteristics of recruited species in rehabilitation trials sites with different soil texture.

\begin{tabular}{|c|c|c|c|c|c|c|}
\hline Especie & $\begin{array}{l}\text { Forma de } \\
\text { crecimiento }\end{array}$ & $\begin{array}{c}\text { Ciclo de } \\
\text { vida }\end{array}$ & Valor de uso & Franco & $\begin{array}{l}\text { Sitio } \\
\text { Franco- } \\
\text { arenoso }\end{array}$ & Arenoso \\
\hline Acantholippia seriphioides (A. Gray) Moldenke & Leñosa & Perenne & No forrajera & & & * \\
\hline Bromus catharticus Vahl. & Herbácea & Anual & Forrajera & & & * \\
\hline Chuquiraga erinacea D. Don. & Leñosa & Perenne & No forrajera & & & * \\
\hline Daucus puscillus Michx. & Herbácea & Anual & Forrajera & & $*$ & \\
\hline Diplotaxis tenuifolia (L.) DC. & Herbácea & Perenne & No forrajera & * & * & \\
\hline Ephedra ochreata Miers & Leñosa & Perenne & Forrajera & & * & \\
\hline Erodium cicutarium (L.) L'Hér. ex Aiton & Herbácea & Anual & Forrajera & * & & \\
\hline Hirschfeldia incana (L.) Lagr.-Foss. & Herbácea & Perenne & No forrajera & * & $*$ & \\
\hline Hoffmannseggia erecta Phil. & Herbácea & Anual & No forrajera & & * & \\
\hline Larrea divaricata Cav. & Leñosa & Perenne & No forrajera & & & * \\
\hline Lycium chilense Miers ex Bertero & Leñosa & Perenne & Forrajera & $*$ & & $*$ \\
\hline Medicago minima (L.) Bartal. & Herbácea & Anual & Forrajera & * & $*$ & \\
\hline $\begin{array}{l}\text { Nardophyllum chiliotrichioides (J. Remy) A. } \\
\text { Gray }\end{array}$ & Leñosa & Perenne & No forrajera & * & & \\
\hline Nassella longiglumis (Phil.) Barkworth & Herbácea & Perenne & Forrajera & $*$ & * & $*$ \\
\hline Nassella tenuis (Phil.) Barkworth & Herbácea & Perenne & Forrajera & * & * & * \\
\hline Pappophorum caespitosum R.E. Fr. & Herbácea & Perenne & Forrajera & * & * & * \\
\hline $\begin{array}{l}\text { Pappostipa speciosa (Trin. and Rupr.) } \\
\text { Romasch. }\end{array}$ & Herbácea & Perenne & No forrajera & * & * & \\
\hline Piptochaetium napostaense (Speg.) Hack. & Herbácea & Perenne & Forrajera & & * & * \\
\hline Plantago patagonica Jacq. & Herbácea & Anual & No forrajera & & * & \\
\hline $\begin{array}{l}\text { Prosopidastrum globosum (Gillies ex Hook. } \\
\text { and Arn.) Burkart }\end{array}$ & Leñosa & Perenne & No forrajera & $*$ & & \\
\hline Salsola kali $\mathrm{L}$ & Herbácea & Anual & No forrajera & * & * & * \\
\hline Schismus barbatus (L.) Thell. & Herbácea & Anual & Forrajera & * & * & * \\
\hline Senecio filaginoides DC. & Leñosa & Perenne & No forrajera & * & $*$ & \\
\hline Solanum sisymbriifolium Lam. & Herbácea & Perenne & No forrajera & * & & \\
\hline
\end{tabular}

Tabla 3. Número de individuos sobrevivientes luego de cuatro años del trasplante. Entre paréntesis se indica el desvío estándar.

Table 3. Number of surviving individuals four years after transplantation. Standard deviation is indicated in parentheses.

\begin{tabular}{lcccc}
\hline & \multicolumn{5}{c}{ Textura del suelo } \\
Especie & Franca & Franco- arenosa & Arenosa & Número inicial por sitio \\
\hline Condalia microphylla & $3( \pm 0.7)$ & $1( \pm 1.3)$ & $2( \pm 3.7)$ & 15 \\
Prosopis flexuosa & $17( \pm 4.8)$ & $21( \pm 2.7)$ & $12( \pm 11.8)$ & 27 \\
Schinus johnstonii & $12( \pm 2.4)$ & $26( \pm 1.6)$ & $8( \pm 7.2)$ & 27 \\
Geoffroea decorticans & $7( \pm 0.8)$ & $13( \pm 6.4)$ & $4( \pm 6.7)$ & 27 \\
Nassella longiglumis & $18( \pm 5.6)$ & $30( \pm 1.3)$ & $9( \pm 7.6)$ & 33 \\
Piptochaetium napostaense & $24( \pm 5.9)$ & $18( \pm 7.5)$ & $12( \pm 10.5)$ & 33 \\
Poppophorum caespitosum & $10( \pm 2.3)$ & $18( \pm 4.9)$ & $8( \pm 9.2)$ & 33 \\
Nassella tenuis & $19( \pm 6.9)$ & $18( \pm 5.6)$ & $14( \pm 12.5)$ & 33 \\
\hline
\end{tabular}


franco-arenoso (95\%) fue mayor que la de $C$. microphylla y $G$. decorticans en los tres sitios considerados (entre 5 y $20 \%$ y entre 15 y $47 \%$, respectivamente). La supervivencia de las especies herbáceas no presentó diferencias significativas entre especies $(\mathrm{F}=1.58 ; \mathrm{P}=0.21)$, sitios $(\mathrm{F}=5.99 ; \mathrm{P}=0.078)$ ni en la interacción entre especies y sitios ( $\mathrm{F}=1.31 ; \mathrm{P}=0.28$ ) (Figura 5).

\section{Composición florística del reclutamiento}

En los sitios de ensayo se registraron 24 especies reclutadas espontáneamente, de las cuales 22 fueron nativas (Tabla 2). La riqueza específica total de individuos de especies leñosas $(\mathrm{H}=3.1 ; \mathrm{P}=0.15)$ y de especies herbáceas $(\mathrm{H}=3.2 ; \mathrm{P}=0.17)$ no presentaron diferencias entre sitios. Al evaluar la riqueza de especies reclutadas, según las distintas categorías establecidas (i.e., herbáceas anuales forrajeras, herbáceas anuales no forrajeras, herbáceas perennes forrajeras, herbáceas perennes no forrajeras, leñosas forrajeras y leñosas no forrajeras) sólo se observaron diferencias significativas entre sitios en la riqueza de especies herbáceas anuales forrajeras $(\mathrm{H}=4.8$; $\mathrm{P}=0.04)$. Estas especies estuvieron presentes en menor medida en el sitio con suelos de textura arenosa en relación con los otros dos sitios. Por otra parte, las especies leñosas forrajeras estuvieron ausentes en el sitio con suelo de textura franco-arenosa (Figura 6). La composición del reclutamiento de herbáceas perennes se correspondió con las especies trasplantadas en los ensayos de rehabilitación.

\section{Densidad de individuos reclutados}

En total, sin discriminar por categoría, la densidad de especies leñosas reclutadas presentó diferencias significativas entre los sitios de ensayo $(\mathrm{H}=0.61, \mathrm{P}=0.027)$ y varió entre 44 individuos $/ \mathrm{m}^{2}$ en el sitio con suelos de textura arenosa y 101 individuos $/ \mathrm{m}^{2}$ en el sitio con suelo de textura franco-arenosa. En cambio, cuando se analizó por separado el reclutamiento de las leñosas forrajeras y no forrajeras, las diferencias entre sitios fueron no significativas $(\mathrm{H}=1.05, \mathrm{P}=0.47$ y $\mathrm{H}=4.89, \mathrm{P}=0.07$, respectivamente) (Figura 7 ).

El análisis del reclutamiento de las herbáceas mostró que la densidad total, sin discriminar por categoría, fue significativamente diferente entre sitios $(\mathrm{H}=1.94 ; \mathrm{P}=0.036)$ y varió entre 56 individuos $/ \mathrm{m}^{2}$ en el sitio de textura arenosa, 219 individuos $/ \mathrm{m}^{2}$ en el sitio de textura francoarenosa y 263 individuos $/ \mathrm{m}^{2}$ en el sitio de textura franca. Cuando se analizaron por separado las distintas categorías distinguidas, se encontró una diferencia significativa en la densidad del reclutamiento en las especies herbáceas anuales forrajeras, que varió entre 12 individuos $/ \mathrm{m}^{2}$ en el sitio con suelo francoarenoso y 194 individuos $/ \mathrm{m}^{2}$ en el sitio con suelo franco $(\mathrm{H}=5.79, \mathrm{P}=0.024)$. La densidad

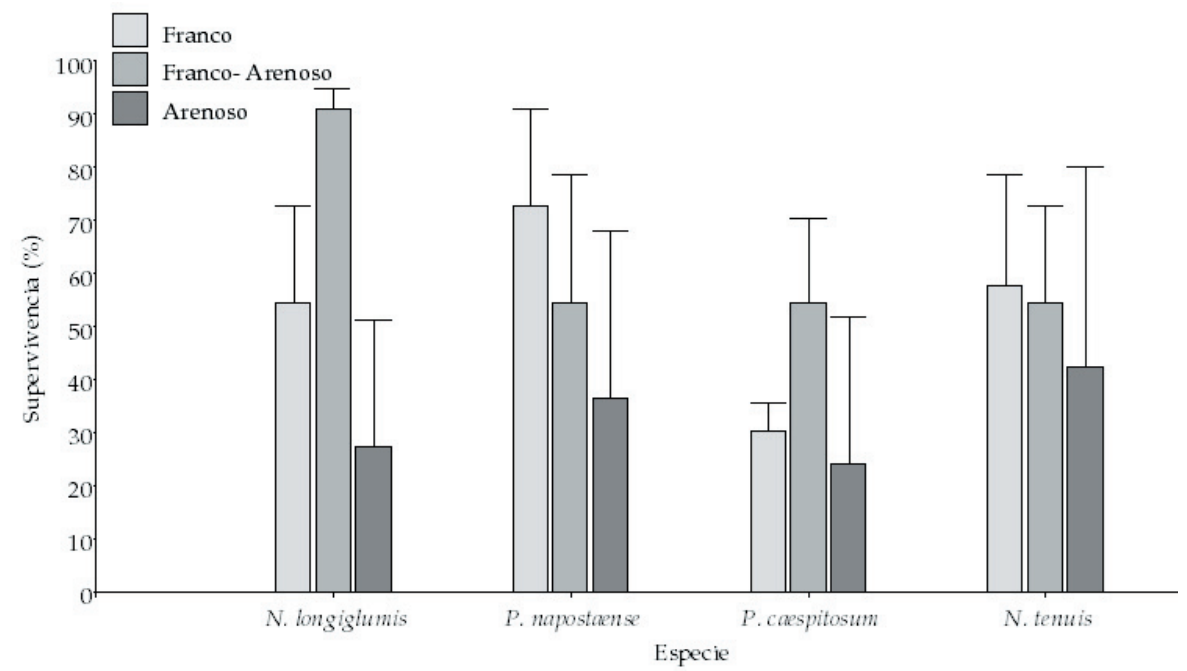

Figura 5. Supervivencia de los individuos de especies herbáceas (Nassella longiglumis, Piptochaetium napostaense, Pappophorum caespitosum y Nassella tenuis) luego de 4 años desde el trasplante. No se detectaron diferencias significativas entre especies, sitios ni en la interacción especies x sitio $(\mathrm{P}<0.05)$. Las barras de error representan el desvío estándar.

Figure 5. Survival of individuals of herbaceous species (Nassella longiglumis, Piptochaetium napostaense, Pappophorum caespitosum and Nassella tenuis) after 4 years from transplantation. Significant differences were not detected among species, sites, neither species $x$ site interaction $(\mathrm{P}<0.05)$. Error bars represent the standard deviation. 


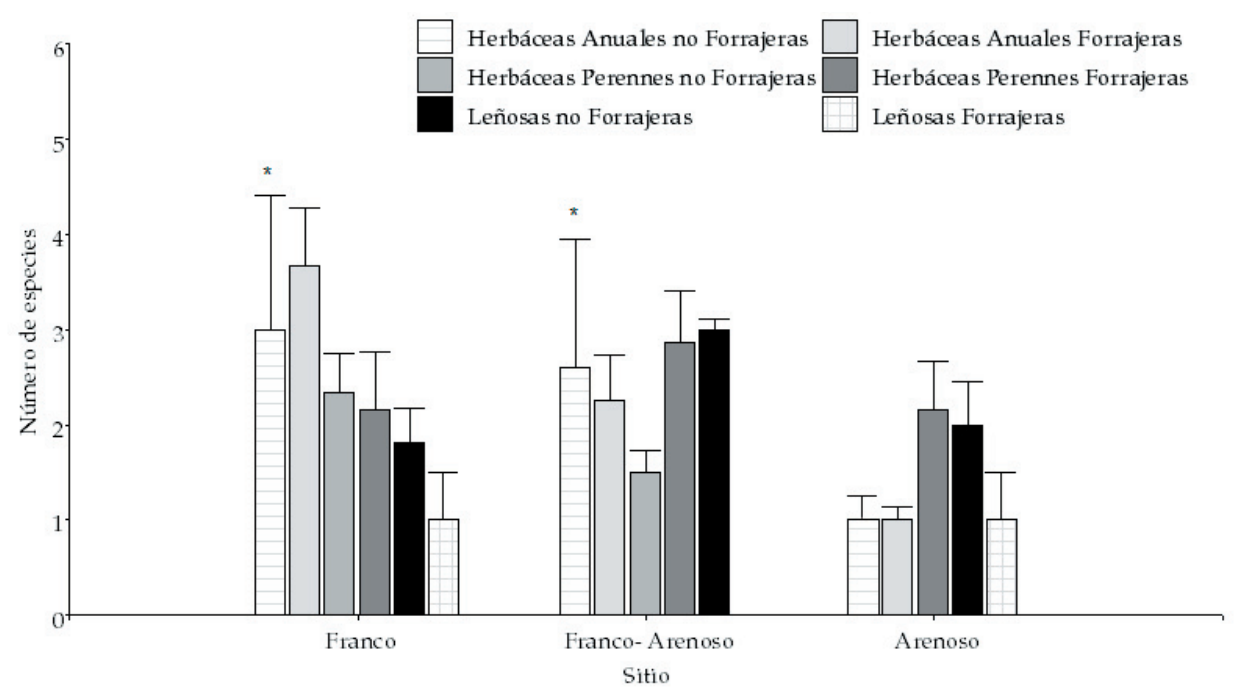

Figura 6. Riqueza específica (número de especies) de plantas herbáceas anuales forrajeras, herbáceas anuales no forrajeras, herbáceas perennes forrajeras, herbáceas perennes no forrajeras, leñosas forrajeras y leñosas no forrajeras en sitios con diferente textura del suelo. El asterisco indica diferencias significativas entre sitios para una misma categoría de plantas $(\mathrm{P}<0.05)$. Las barras de error representan el desvío estándar.

Figure 6. Species richness (number of species) of herbaceous forage annual, non-forage annual, perennial forage, perennial non-forage, woody forage, and woody non-forage plants in sites with different soil texture. Asterisk indicates significant differences between sites for the same plant category $(\mathrm{P}<0.05)$. Error bars represent the standard deviation.

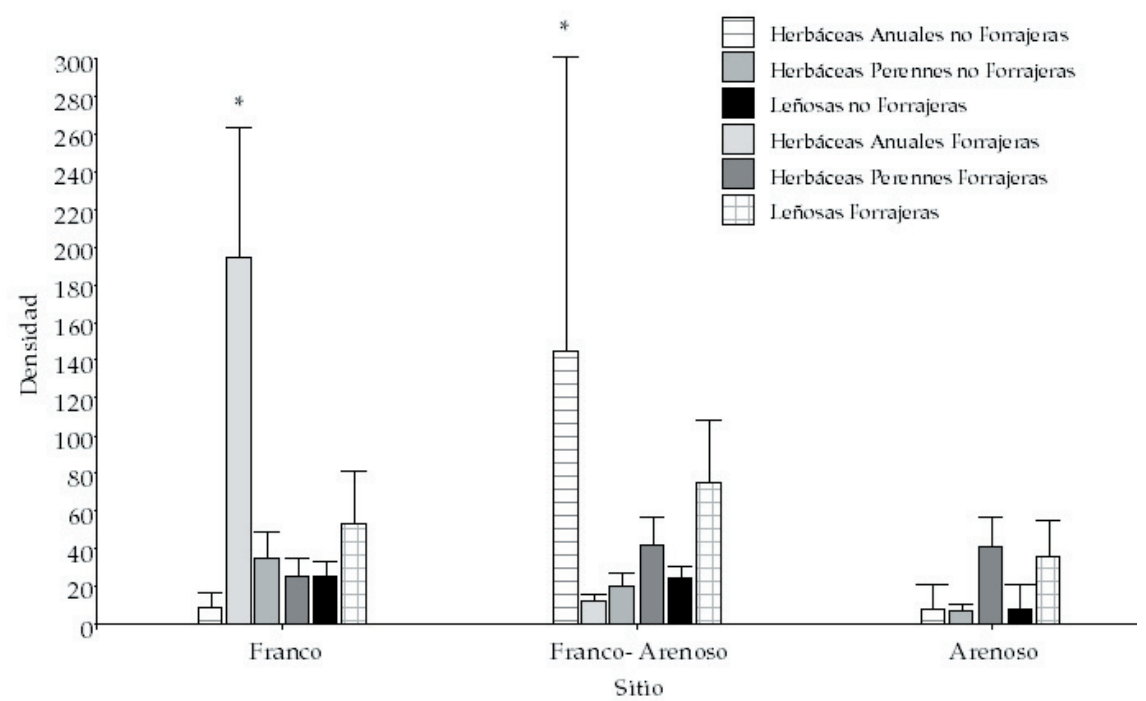

Figura 7. Densidad (individuos $/ \mathrm{m}^{2}$ ) de plantas herbáceas anuales forrajeras, herbáceas anuales no forrajeras, herbáceas perennes forrajeras, herbáceas perennes no forrajeras, leñosas forrajeras y leñosas no forrajeras en sitios con diferente textura del suelo. El asterisco indica diferencias significativas entre sitios para una misma categoría de plantas $(\mathrm{P}<0.05)$. Las barras de error representan el desvío estándar.

Figure 7. Density (individuals $/ \mathrm{m}^{2}$ ) of herbaceous forage annual, non-forage herbaceous annual, perennial herbaceous forage, perennial herbaceous non-forage, woody forage, and woody non-forage plants in sites with different soil texture. Asterisk indicates significant differences between sites for the same plant category $(\mathrm{P}<0.05)$. Error bars represent the standard deviation.

de especies herbáceas anuales no forrajeras también fue diferente según el sitio $(\mathrm{H}=10.6$, $\mathrm{P}=0.004)$. La mayor densidad de esta categoría se observó en el sitio con suelo franco-arenoso (145 individuos $/ \mathrm{m}^{2}$ ), seguido por los sitios con suelos de textura franca y arenosa, con 9 y 8 individuos $/ \mathrm{m}^{2}$, respectivamente. La densidad del reclutamiento de las especies herbáceas perennes forrajeras varió, según el sitio, entre 42 y 25 individuos $/ \mathrm{m}^{2}(\mathrm{H}=0.09, \mathrm{P}=0.95)$, mientras que la densidad del reclutamiento de las especies herbáceas perennes no forrajeras varió entre 7 y 35 individuos $/ \mathrm{m}^{2}(\mathrm{H}=2.4$, $\mathrm{P}=0.29$ ) (Figura 7). 


\section{Discusión}

La supervivencia de las especies empleadas en los ensayos de rehabilitación ecológica no mostró una clara relación con la textura del suelo. Se esperaba que el sitio con suelos de textura franca presentara mayores porcentajes de supervivencia de los individuos trasplantados que los sitios con suelos con mayor contenido de arena, en relación con sus mejores características granulométricas para retener humedad. Sin embargo, la mayoría de las especies del ensayo no mostraron diferencias significativas en su supervivencia entre los sitios considerados, excepto $S$. johnstonii en suelos de textura franco-arenosa. Al contrario de lo esperado, en el caso de $S$. johnstonii se observó una menor supervivencia en el sitio de suelo franco, comparado con el sitio de suelo franco-arenoso. La misma tendencia fue observada en el caso de $P$. flexuosa y G. decorticans. Este sitio reuniría condiciones relativamente favorables en cuanto a su capacidad de retención de agua, comparado con el sitio arenoso y, además, un desarrollo en profundidad mayor que el sitio de textura franca. Este último presenta limitaciones severas en su profundidad debido a la pérdida de los horizontes superficiales y a la escasa profundidad del suelo hasta el contacto con una capa homogénea de $\mathrm{CaCO}_{2}$. Esta capa, que se halla situada a solo $15 \mathrm{~cm}$ de profundidad, ofrece una barrera potente para el desarrollo del sistema radical más profundo de los ejemplares de las especies leñosas. La degradación de este sitio está asociada a su historia de uso. Fue desmontado a tala rasa, se lo destinó al uso agrícola y luego se lo abandonó sin mediar ninguna estrategia de manejo durante los años en que la sequía afectó fuertemente el área (Zeberio 2012). Los procesos de degradación causaron la pérdida de la capa de suelo superficial, dejando a escasa profundidad una capa homogénea de carbonato de calcio. La presencia de esta capa ofrece una barrera al desarrollo radical profundo de los individuos, principalmente de especies leñosas. Zeberio y Pérez (2020) observaron que la profundidad del suelo es una de las características edáficas que determinan la supervivencia de estas especies leñosas nativas en el área de estudio. Las especies leñosas de la Patagonia poseen sistemas de raíces profundas que le permiten captar un mayor volumen de recursos (Bucci et al. 2011; Jobbágy et al. 2011; Villagra et al. 2009). Schinus johnstonii y P. flexuosa poseen un sistema radical dimórfico que les permite explorar las capas más profundas del suelo e independizarse de las precipitaciones. Además, con la porción más somera de sus raíces aprovechan la humedad que las lluvias recientes aportan a las capas más superficiales (Bucci et al. 2009; Jobbágy et al. 2011; Villagra 2000; Villagra et al. 2011). Esta característica del sistema subterráneo está ausente en las otras especies consideradas en este estudio. Peláez et al. (1994) indican que C. microphylla posee un sistema radical somero, distribuido en los primeros $60 \mathrm{~cm}$ del perfil del suelo, y compite con los pastos por la humedad que aportan las lluvias. Por otra parte, G. decorticans posee un sistema radical pivotante y profundo, con crecimiento gemífero (Krauss et al. 2003), pero las plantas de esta especie fueron dañadas severamente por la liebre patagónica (Dolichotis patagonum), lo que impide identificar con claridad las causas de su mortalidad elevada. Las clausuras establecidas impidieron el pastoreo de herbívoros domésticos, pero fueron insuficientes para impedir el ingreso de la liebre patagónica, cuya marcada preferencia por $G$. decorticans no fue prevista. Las especies herbáceas trasplantadas mostraron, como tendencia, elevadas supervivencias en los sitios con suelos franco y franco-arenoso, lo que se podría vincular con una mayor capacidad de retención de agua de esos suelos y con el desarrollo de un sistema radical somero de las especies herbáceas, que alcanza su mayor desarrollo en los primeros $20 \mathrm{~cm}$ de profundidad (Soriano et al. 1987). La alta densidad de raíces superficiales le permitiría a las especies herbáceas usar de forma más eficiente los eventos de lluvias de menor volumen, que son los más frecuentes en estos sistemas (Aguiar and Sala 1994; Paruelo and Sala 1995). En los sitios con textura arenosa, el agua de las precipitaciones se pierde por percolación profunda debido a que estos suelos son excesivamente drenados (Godagnone and Bran 2009), lo que reduciría la disponibilidad de agua para la vegetación. Sin embargo, nuestros resultados no admiten a la textura del suelo como factor determinante de la supervivencia de las herbáceas trasplantadas.

La riqueza de especies reclutadas en los ensayos de rehabilitación ecológica no mostró una relación evidente con la textura del suelo, excepto para las herbáceas anuales no forrajeras, que fueron mayores en suelos francos y franco-arenosos. Dado que se señala a la falta de agua en el suelo como el factor limitante más importante en el establecimiento 
de la vegetación en el noreste de la Patagonia (Gaitán et al. 2008), se esperaba que este factor tuviese preponderancia sobre otros. Sin embargo, los resultados obtenidos no permiten sostener nuestra hipótesis. Otros factores estarían ejerciendo una marcada influencia en la composición florística y riqueza del reclutamiento en cada sitio. Entre esos factores se destaca la disponibilidad de semillas. En áreas degradadas del Monte patagónico, Bisigato y Bertiller (2004) señalan que el reclutamiento de pastos perennes está condicionado por la distribución espacial del banco de semillas y por la disponibilidad de agua del suelo. En el noreste patagónico, el fuego y el pastoreo afectan negativamente la lluvia de semillas de leñosas arbustivas y pastos perennes (Leder et al. 2015). Los sitios del presente estudio, abandonados luego de varios años de agricultura, estuvieron sometidos a pastoreo por ganado doméstico, sin un apotreramiento que permita descansos y rotación de la carga (Zeberio 2018). A su vez, estudios realizados en la zona analizaron el efecto de distintas intensidades de pastoreo y de sequías prolongadas, y hallaron que las dicotiledóneas herbáceas fueron las más abundantes en los bancos de semillas analizados, lo que se relaciona con los efectivos mecanismos de dispersión de sus semillas y con el efecto del pastoreo, que tiende a favorecer la abundancia de plantas de ese grupo funcional (Funk et al. 2019). En ese sentido, nuestros resultados también muestran un predominio en el reclutamiento de las herbáceas anuales (i.e., forrajeras y no forrajeras), la mayoría de ellas dicotiledóneas.

La historia de uso de los sitios de estudio habría tenido un efecto negativo sobre la disponibilidad de semillas de las especies perennes, tanto leñosas como herbáceas. A esto se debería sumar que las especies anuales suelen ser dominantes en áreas afectadas por disturbios debido a que sus cortos ciclos de vida les permiten desarrollarse en períodos térmica e hídricamente favorables, y alcanzar tempranamente su madurez; además, producen una elevada cantidad de semillas de pequeño tamaño, con alta capacidad de dispersión y morfologías que facilitan su entrada en el banco de semillas del suelo (Fenner and Thompson 2005; Venable et al. 2008; Franzese et al. 2015). Sin embargo, y a pesar del elevado número de individuos que registramos en el reclutamiento, su rol en la rehabilitación de ecosistemas es menos valorado que el de las especies perennes porque ofrecen cobertura al suelo durante un período breve del año y no son una fuente de forraje importante debido al escaso volumen de biomasa que representan (Pucheta et al. 2011).

En el presente estudio no se registró reclutamiento de las especies leñosas trasplantadas. Los sitios de este estudio presentaban porcentajes de suelo desnudo $>90 \%$ al momento de instalar las parcelas de ensayo, producto de su historia de uso (Zeberio 2018). Posiblemente, la ausencia de un banco de semillas viable y de fuentes de semillas en las cercanías de estos sitios sean las causantes de la ausencia de reclutamiento de esas leñosas, ya que los ejemplares trasplantados no fructificaron en el período de mediciones. En cambio, la composición específica del reclutamiento de herbáceas perennes estuvo determinada por las especies empleadas en los ensayos de rehabilitación, que generaron estructuras reproductivas en el período de estudios. En contraposición a la estrategia de las especies anuales, las especies herbáceas perennes generalmente presentan semillas de mayor tamaño y peso que no pueden ser transportadas a grandes distancias, generan un menor número de semillas y no están sujetas a los pulsos ambientales (Distel et al. 1992). Desde el punto de vista ecológico, estas especies son importantes porque protegen el suelo de los factores erosivos del clima y por ser una fuente de forraje que, manejada correctamente, permite alcanzar la sustentabilidad de los sistemas pastoriles (Morici et al. 2006). Bisigato y Bertiller (1999; 2004), en el Monte de la provincia de Chubut, observaron mayores porcentajes de establecimiento y supervivencia de plántulas de pastos perennes en sitios cercanos a los ejemplares adultos de esas especies $y$, en menor medida, de especies leñosas, que actuaron como facilitadoras. La existencia de mecanismos facilitadores en la Patagonia fue reportada en numerosos estudios (Raffaele and Veblen 1998; Kitzberger et al. 2000; Valenzuela et al. 2016; González and Pérez 2017). Si bien no se conoce que se hayan desarrollado este tipo de trabajos para las especies presentes en el noreste de la Patagonia, sería deseable seguir reuniendo evidencia que permita profundizar sobre los procesos de facilitación, a fin de emplear de forma más eficiente a las especies nativas perennes en trabajos de rehabilitación ecológica. 


\section{ConClusión}

Este trabajo permitió identificar a las herbáceas perennes forrajeras $N$. tenuis, $N$. longiglumis, $P$. napostaense y $P$. caespitosum, y a las leñosas $S$. johnstonii y P. flexuosa como especies con buenas perspectivas para proyectos de rehabilitación ecológica de áreas degradadas en el Monte patagónico, por su elevada supervivencia, y en el caso de los pastos perennes, también por su aporte al repoblamiento con esas especies deseables por su valor forrajero y su cobertura del suelo. Los resultados obtenidos no permitieron asignar a la textura del suelo, en relación con su capacidad de retención de agua, un efecto determinante tanto sobre la supervivencia de los ejemplares trasplantados como sobre el reclutamiento de diversas categorías de plantas. Futuros estudios deberían profundizar la interacción entre las características edáficas de áreas degradadas, la provisión de semillas desde diversas fuentes y la acción facilitadora de las especies nativas sobre la recuperación de la vegetación.

Agradecimientos. El trabajo fue financiado por la Universidad Nacional de Río Negro (PI 40-C-355) y por el Ministerio Nacional de Ciencia y Técnica (PICTO 2010/0219). Agradecemos a los revisores anónimos y a la Dra. María Vanessa Lencinas por sus valiosas sugerencias y comentarios que permitieron mejorar el manuscrito.

\section{REFERENCIAS}

Abraham, E. M., J. C. Guevara, R. J. Candia, and N. D. Soria. 2016. Dust storm, drought and desertification in the Southwest of Buenos Aires Province, Argentina. Revista de la Facultad de Ciencias Agropecuarias de la Universidad Nacional de Cuyo 48:221-241.

Aguiar, M. R., and O. E. Sala. 1994. Competition, facilitation, seed distribution and the origin of patches in a Patagonian steppe. Oikos 70:26-34. https://doi.org/10.2307/3545695.

Aguiar, M. R., and O. E. Sala. 1999. Patch structure, dynamics and implications for the functioning of arid ecosystems. Trends in Ecology and Evolution 14:273-277. https://doi.org/10.1016/S0169-5347(99)01612-2.

Aronson, J., C. Floret, E. LeFloc'h, C. Ovalle, and R. Pontainer. 1993. Restoration and rehabilitation of degrades ecosystems in arid and semi arid lands. I. a view from the south. Restoration Ecology 1:8-17. https://doi.org/10.1111/ j.1526-100X.1993.tb00004.x.

Bisigato, A. J., and M. B. Bertiller. 1999. Seedling emergence and survival in contrasting soil microsites in Patagonian Monte shrubland. Journal of Vegetation Science 10:335-342. https://doi.org/10.2307/3237062.

Bisigato, A. J., and M. B. Bertiller. 2004. Seedling recruitment of perennial grasses in degraded areas of the Patagonian Monte. Journal of Range Management 57:191-196. https://doi.org/10.2307/4003918.

Bucci, S. J., F. G. Scholtz, G. Goldstein, F. C. Meinzer, and M. E. Arce. 2009. Soil water availability and rooting depth as determinants of hydraulic architecture of Patagonian woody species. Oecología 160:631-641.

Bucci, S. J., F. G. Scholz, P. A. Iogna, and G. Goldstein. 2011. Economía del agua de especies arbustivas de las estepas patagónicas. Ecología Austral 21:43-60.

Buschiazzo, D. E., A. R. Quiroga, and K. Stahr. 1991. Patterns of organic matter distribution in soils of the semiarid Argentinian Pampas. Pflanzenernah 154:437-441. https://doi.org/10.1002/jpln.19911540608.

Buschiazzo, D. E., H. D. Esterlich, S. B. Aimar, E. Viglizzo, and J. Babinec. 2004. Soil texture and tree coverage influence on organic matter. Journal of Range Management 57:511-516. https://doi.org/10.2307/4003981.

Conover, W.J. 1999. Practical Nonparametric Statistics. John Wiley \& Sons, Inc., New York.

Conover, W. J. and R. L. Inman. 1979. On multiple comparison procedures. Los Alamos Sci. Lab. Tech. Rep. Ed. Los Angeles.

Cortina, J., B. Amat, V. Castillo, D. Fuentes, F. T. Maestre, F. M. Padilla, and L. Rojo. 2011. The restoration of vegetation cover in the semiarid Iberian southeast. Journal of Arid Environments 75:377-1384. https://doi.org/10.1016/ j.jaridenv.2011.08.003.

Dalmaso, A. D. 2010. Revegetación de áreas degradadas con especies nativas. Boletín de la Sociedad Argentina de Botánica 45:149-171.

De Berasategui, L. 2004. Estadísticas climáticas del valle de Viedma- 30 años. Boletín de Información Técnica №20. EEA Valle Inferior. Ediciones INTA.

Delgado-Baquerizo, M., F. T. Maestre, A. Gallardo, M. A. Bowker, M. D. Wallenstein, J. L. Quero, V. Ochoa, B. Gozalo, M. García-Gómez, S. Soliveres, P. García-Palacios, M. Berdugo, E. Valencia, C. Escolar, T. Arredondo, C. BarrazaZepeda, D. Bran, J. A. Carreira, M. Chaieb, A. A. Conceiçao, M. Derak, D. J. Eldridge, A. Escudero, C. I. Espinosa, J. J. Gaitán, M. G. Gatica, S. Gómez-González, E. Guzman, J. R. Gutierrez, A. Florentino, E. Hepper, R. M. Hernández, E. Huber-Sannwald, M. Jankju, J. Liu, R. L. Mau, M. Miriti, J. Monerris, K. Naseri, Z. Noumi, V. Polo, A. Prina, E. Pucheta, E. Ramírez, D. A. Ramírez-Collantes, R. Romao, M. Tighe, D. Torres, C. Torres-Dýaz, E. D. Ungar, J. Val, W. Wamiti, D. Wang, and E. Zaady. 2013. Decoupling of soil nutrient cycles as a function of aridity in global drylands. Nature 502:672-670. https://doi.org/10.1038/nature12670.

Di Rienzo, J. A., F. Casanoves, M. Balzarini, L. González, E. Tablada, and C. Robledo. 2018. Centro de Transferencia InfoStat, FCA, Universidad Nacional de Córdoba, Argentina. 
Distel, R. A., D. V. Peláez, and O. A. Fernández. 1992. Germination of Piptochaetium napostaense (Speg.) Hackel and Stipa tenuis Phil. and seedling survival under field conditions. Rangeland Journal 14:49-55.

Faraway, J. J. 2006. Extending the Linear Model With R: Generalized Linear, Mixed Effects and Nonparametric Regression Models. Chapman and Hall, Boca Raton, Florida, USA.

Fenner, M., and K. Thompson. 2005. The ecology of seeds. Cambridge University Press, Cambridge, UK.

Franzese, J., L. Ghermandi, and S. L. González. 2015. Historical land use by domestic grazing revealed by the soil seed bank: a case study from a natural semi-arid grassland of NW Patagonia. Grass and Forage Science 70:1-13.

Funk, F. A., Loydi, A., Peter, G., and R. A. Distel. 2019. Effect of grazing and drought on seed bank in semiarid patchy rangelands of northern Patagonia, Argentina. International Journal of Plant Sciences 180:337-344. https://doi.org/ $10.1086 / 702661$.

Gaitán, J. J., C. R. López, and D. E. Bran. 2008. Grazing effects on soil and vegetation in the Patagonian steppe. Ecología Austral 27:1-10.

Gasch, C., S. Huzurbazar, and P. Stahl. 2014. Measuring soil disturbance effects and assessing soil respiration success by examining distribution of soil properties. Applied Soil Ecology 76:102-111. https://doi.org/10.1016/ j.apsoil.2013.12.012.

Godagnone, R. E., and D. E. Bran 2009. Inventario integrado de los recursos naturales de la Provincia de Río Negro. INTA, Buenos Aires, Argentina.

González, F. M., and D. R. Pérez. 2017. Contributions of ecological facilitation for restoring environments with high conservation value in the Argentine Patagonia. Phyton 86:332-339. https://doi.org/10.32604/phyton.2017.86.332.

Hobbs, R., and V. Cramer. 2008. Restoration ecology: Interventionist approaches for restoring and maintaining ecosystems function in the face of rapid environmental change. Annual Review of Environment and Resources 33: 39-61. https://doi.org/10.1146/annurev.environ.33.020107.113631.

Jobbágy, E. G., M. D. Nosetto, P. E. Villagra, and R. B. Jackson. 2011. Water subsidies from mountains to deserts: Their role in sustaining groundwater- fed oases in a sandy landscape. Ecological Applications 21:678-694. https: //doi.org/10.1890/09-1427.1.

Kitzberger, T., D. F. Steinaker, and T. T Veblen. 2000. Effects of climatic variability on facilitation of tree establishment in northern Patagonia. Ecology 81:914-1924. https://doi.org/10.1890/0012-9658(2000)081[1914:EOCVOF]2.0.CO;2.

Krauss, T., C. Bianco, and F. Weberling. 2003. Root system morphology of Fabaceae species from central Argentina. Wulfenia 10:61-72.

Kröpfl, A. I., N. M. Villasuso, and G. Peter. 2012. Guía para el reconocimiento de especies de los pastizales del Monte Oriental de Patagonia. INTA, Bariloche, Argentina.

Landi, M., and D. Renison. 2010. Forestación con Polylepis australis BITT. En suelos erosionados de las Sierras Grandes de Córdoba. Ecología Austral 20:47-55.

Leder, C. V., Peter, G., and F. A. Funk. 2015. Seed rain alteration related to fire and grazing history in a semiarid shrubland. Journal of Arid Environments 121:32-39. https://doi.org/10.1016/j.jaridenv.2015.05.012.

Le Houerou, H. N. 1989. The grazing land ecosystems of the Africa Sahel. Ecological Studies vol. 75. Springer, Berlin.

León, R. J., D. E. Bran, M. Collantes, J. M. Paruelo, and A. Soriano. 1998. Grandes unidades de vegetación de la Patagonia extra andina. Ecología Austral 8:125-144.

López Lauenstein, D., M. E. Fernández, and A. Verga. 2012. Respuesta diferenciada a la sequía de plantas jóvenes de Prosopis chilensis, P. flexuosa y sus híbridos interespecíficos: implicancias para la reforestación en zonas áridas. Ecología Austral 22:43-52.

Maestre, F. T., J. Cortina, S. Bautista, J. Bellot, and R. Vallejo. 2003. Small- scale environmental heterogeneity and spatiotemporal dynamics of seedlings establishment in a semiarid degraded ecosystem. Ecosystems 6:630-643. https: //doi.org/10.1007/s10021-002-0222-5.

Maestre, F. T., S. Bautista, J. Cortina, and J. Bellot. 2001. Potential for using facilitation by grasses to establish shrubs on a semiarid degraded steppe. Ecological Applications 11:1641-1655. https://doi.org/10.1890/1051-0761(2001)011[1641: PFUFBG]2.0.CO;2.

Maestre, F. T., D. J. Eldridge, S. Soliveres, S. Kefi, M. Delgado-Baquerizo, M. Bowker, P. García- Palacios, J. J. Gaitán, A. Gallardo, R. Lázaro, and M. Berdugo. 2016. Structure and functioning of drylands ecosystems in a changing world. Annual Review of Ecology, Evolution and Systematics 47:215-237. https://doi.org/10.1146/annurev-ecolsys121415-032311.

Morello, J., S. Matteucci, A. F. Rodríguez, and M. Silva. 2012. Ecorregiones y complejos ecosistémicos argentinos. Orientación Gráfica Editora, Buenos Aires, Argentina.

Morici, E., W. Muiño, R. Ernst, and S. Poey. 2006. Efecto de la distancia a la aguada sobre la estructura del estrato herbáceo en matorrales de Larrea sp. Pastoreados por bovinos en zonas áridas de Argentina. Archivos de Zootecnia 55:149-159.

Padilla, F. M., and F. I. Pugnaire. 2006. The role of nurse plants in the restoration of degraded environments. Frontiers in Ecology and the Environment 4:196-202. https://doi.org/10.1890/1540-9295(2006)004[0196:TRONPI]2.0.CO;2.

Paruelo, J. M., and O. E. Sala. 1995. Water losses in the Patagonian steppe: a modelling approach. Ecology 76:510-520. https://doi.org/10.2307/1941209.

Peláez, D. V., R. Distel, R. Bóo, O. R. Elia, and M. D. Mayor. 1994. Water relations between shrubs and grasses in semiarid Argentina. Journal of Arid Environments 27:71-78. https://doi.org/10.1006/jare.1994.1046. 
Peláez, D. V., R. Bóo, and O. R. Elia. 1996. The germination and seedling survival of Condalia microphylla Cav. in Argentina. Journal of Arid Environments 32:173-179. https://doi.org/10.1006/jare.1996.0015.

Plaza Behr, M. C., C. A. Pérez, J. F. Goya, M. Azcona, and M. F. Arturi. 2016. Plantación de Celtis ehrenbergiana como técnica de recuperación de bosques invadidos por Ligustrum lucidum en los talares del NE de Buenos Aires. Ecología Austral 26:171-177. https://doi.org/10.25260/EA.16.26.2.0.176.

Pucheta, E., V. J. García Muro, A. G. Rolhauser, and L. Quevedo- Robledo. 2011 Invasive potential of the winter grass Schismus barbatus during the winter season of predominantly summer-rainfall desert in Central-Northern Monte. Journal of Arid Environments 75:390-393.

Raffaele, E., and T. T. Veblen. 1998. Facilitation by nurse shrubs of resprouting behavior in a post-fire shrubland in northern Patagonia, Argentina. Journal of Vegetation Science 9:693-698. https://doi.org/10.2307/3237287.

Seghieri, J., S. Galle, J. L. Rajot, and M. Ehrmann. 1997. Relations between soil moisture and growth of herbaceous plants in a natural vegetation mosaic in Niger. Journal of Arid Environments 36:87-102. https://doi.org/10.1006/ jare.1996.0195.

Soriano, A., R. A. Golluscio, and E. H. Satorre. 1987. Spatial heterogeneity of the root system of grasses in the Patagonian arid steppe. Bulletin of the Torrey Botanical Club 114:103 108.

Tongway, D. J., and N. L. Hindley. 2005. Landscape function analysis: Procedures for monitoring and assessing landscapes. CSIRO, Melbourne, Australia.

Torres, R. C., M. Giorgis, and C. Trillo. 2015. Supervivencia y crecimiento de especies con distinta estrategia de vida en plantaciones de áreas quemadas y no quemadas: un estudio de caso con dos especies leñosas en el Chaco Serrano, Argentina. Ecología Austral 25:135-143. https://doi.org/10.25260/EA.15.25.2.0.158.

Torres Robles, S. S., M. F. Arturi, C. Contreras, G. Peter, and J. M. Zeberio. 2015. Variaciones geográficas de la estructura y composición de la vegetación leñosa en el límite entre el Espinal y el Monte en el noreste de la Patagonia. Boletín de la Sociedad Argentina de Botánica 50:209-215. https://doi.org/10.31055/1851.2372.v50.n2.11664.

Valentin, C., and L. M. Bresson. 1992. Morphology, genesis and classification of soil crusts in loamy and sandy soils. Geoderma 55:225-245. https://doi.org/10.1016/0016-7061(92)90085-L.

Valenzuela, P., E. C. Arellano, J. A. Burger, and P. Becerra. 2016. Using facilitation microsites as a restoration tool for conversion of degraded grasslands to Nothofagus forests in Southern Patagonia. Ecological Engineering 95:580-587. https://doi.org/10.1016/j.ecoleng.2016.06.116.

Valladares, F., and E. Gianoli. 2007. How Much Ecology Do We Need to Know to Restore Mediterranean Ecosystems? Restoration Ecology 15:363-368.

Venable, D. L., A. Flores-Martínez, H. C. Muller-Landau, G. Barron-Gafford, and J. X. Becerra. 2008. Seed dispersal of desert annuals. Ecology 89:2218-2227. https://doi.org/10.1890/07-0386.1.

Villagra, P. E. 2000. Aspectos ecológicos de los algarrobales argentinos. Multequina 9:35-51.

Villagra, P. E., G. E. Defossé, H. F. del Valle, S. Tabeni, M. Rostagno, E. Cesca, and E. Abraham. 2009. Land use and disturbance effects on the dynamics of natural ecosystems of the Monte Desert: Implications for their management. Journal of Arid Environments 73:202-211. https://doi.org/10.1016/j.jaridenv.2008.08.002.

Villagra, P. E., C. V. Giordano, J. A. Álvarez, J. B. Cavagnaro, A. Guevara, C. Sartor, C. Passera, and S. Greco. 2011. Ser planta en el desierto: estrategias de uso de agua y resistencia al estrés hídrico en el Monte Central de Argentina. Ecología Austral 21:29-42.

Ward, R. C., and M. Robinson. 1990. Principles of Hydrology, Second edition. McGraw-Hill. New York, New York, USA.

Whisenant, S. 1999. Repairing Damaged Wildlands: A Process- Orientated, Landscape-Scale Approach. Cambridge University Press, Cambridge, UK.

Zeberio, J. M. 2012. Avance de la frontera agropecuaria en el noreste patagónico y sus consecuencias en los procesos de desertificación y pérdida de biodiversidad. Pp. 216-221 en M. R. Dos Santos Alfonso (ed.). Ciencia y Tecnología Ambiental. Un Enfoque Integrador. Asociación Argentina para el progreso de la ciencia, Buenos Aires, Argentina.

Zeberio, J. M. 2018. Estado de conservación y posibilidades de rehabilitación en ecosistemas semiáridos: El caso del Monte en el noreste de Río Negro. Tesis doctoral. Facultad de Ciencias Naturales y Museo, Universidad Nacional de La Plata, Buenos Aires. Argentina. Pp. 180.

Zeberio, J. M., S. S. Torres Robles, and G. M. Calabrese. 2018. Uso del suelo y estado de conservación de la vegetación leñosa del monte en el noreste patagónico. Ecología Austral 28:543-552. https://doi.org/10.25260/EA.18.28.3.0.471.

Zeberio, J. M., and C. A. Pérez. 2020. Rehabilitation of degraded areas in northeastern Patagonia, Argentina: effects of environmental condition and plant functional traits on performance of woody vegetation. Journal of Arid Land 12: 653-655. https://doi.org/10.1007/s40333-020-0021-x.

Zuloaga, F. O., E. G. Nicora, Z. E. Rugolo de Agrasar, O. Morrone, J. Pensiero, and M. Cialdella. 1994. Catálogo de la familia Poaceae en la República Argentina. Monography Systematic Botanical Missouri Botanical Garden 47:1-178.

Zuloaga, F. O., and O. Morrone. 1996. Catálogo de las plantas vasculares de la República Argentina I. Pteridophyta, Gymnospermae y Angiospermae (Monocotiledoneae), Monography Systematic Botanical Missouri Botanical Garden 60:1-323.

Zuloaga, F. O., and O. Morrone. 1999. Catálogo de las plantas vasculares de la República Argentina II. AcanthaceaeEuphorbiaceae (Dicotyledoneae), Monography Systematic Botanical Missouri Botanical Garden 74:1-621. 